\title{
Sistematização da assistência de enfermagem à gestante no centro cirúrgico obstétrico: potencialidades e desafios
}

Systematization of nursing care for pregnant women in the obstetric surgical center: potentials and challenges

Sistematización de la atención de enfermería a la gestante en el centro quirúrgico obstétrico: potencialidades y desafíos

\section{Resumo}

Objetivo: Investigar as evidências científicas sobre a assistência de enfermagem à gestante no centro cirúrgico obstétrico instrumentalizada pelo Processo de Enfermagem. Método: Foi realizado uma revisão integrativa da literatura consultando as bases de dados BDENF, LILACS e SciELO através da Biblioteca Virtual em Saúde e site oficial da SciELO. Foram utilizados os descritores controlados e não controlados: centro cirúrgico, gestante, centro cirúrgico obstétrico, gravidez, gestação de alto risco, processo de enfermagem e sistematização da assistência de enfermagem. Para formulação da estratégia de busca, os descritores foram combinados entre si com uso dos operadores booleanos $A N D$ e $O R$. Da elegibilidade, foram incluídos estudos primários, disponíveis online, em português e inglês, considerando o recorte temporal de 2016 a 2020 e excluídas teses, manuais, monografias, dissertações, editoriais, guias de prática clínica e revisões. Resultados: Foram selecionados seis artigos que revelaram que o enfermeiro reconhece a importância da aplicação do processo de enfermagem, porém alega falta de tempo pela sobrecarga de trabalho para sua concretização. Considerações Finais: foi percebido lacuna nas publicações quanto a temática da aplicação da sistematização da assistência de enfermagem no centro cirúrgico obstétrico, fato que corrobora para o fomento de futuros estudos sobre o assunto.

Palavras-chave: Cuidados de enfermagem; Processo de enfermagem; Centro cirúrgico; Assistência de enfermagem; Gravidez. 


\begin{abstract}
Objective: To investigate the scientific evidence on nursing care for pregnant women in the obstetric surgical center instrumented by the Nursing Process. Method: An integrative literature review was carried out by consulting the BDENF, LILACS and SciELO databases through the Virtual Health Library and SciELO's official website. Controlled and uncontrolled descriptors were used: operating room, pregnant woman, obstetric operating room, pregnancy, high-risk pregnancy, nursing process and systematization of nursing care. To formulate the search strategy, the descriptors were combined with each other using the Boolean operators $A N D$ and $O R$. Eligibility included primary studies, available online, in Portuguese and English, considering the time frame from 2016 to 2020 , excluding theses, manuals, monographs, dissertations, editorials, clinical practice guides and reviews. Results: Six articles were selected that revealed that the nurse recognizes the importance of applying the nursing process, but alleges lack of time due to the work overload for its implementation. Final Considerations: a gap was perceived in the publications regarding the theme of applying the systematization of nursing care in the obstetric surgical center, a fact that corroborates the encouragement of future studies on the subject.
\end{abstract}

Keywords: Nursing care; Nursing process; Surgery center; Nursing assistance; Pregnancy.

\title{
Resumen
}

Objetivo: Investigar la evidencia científica sobre la atención de enfermería a la gestante en el centro quirúrgico obstétrico instrumentado por el Proceso de Enfermería. Método: Se realizó una revisión integradora de la literatura mediante la consulta de las bases de datos BDENF, LILACS y SciELO a través de la Biblioteca Virtual en Salud y el sitio web oficial de SciELO. Se utilizaron descriptores controlados y no controlados: quirófano, gestante, quirófano obstétrico, embarazo, embarazo de alto riesgo, proceso de enfermería y sistematización de los cuidados de enfermería. Para formular la estrategia de búsqueda, los descriptores se combinaron entre sí utilizando los operadores booleanos $A N D$ y $O R$. La elegibilidad incluyó estudios primarios, disponibles en línea, en portugués e inglés, considerando el período de 2016 a 2020, excluyendo tesis, manuales, monografías, disertaciones, editoriales, guías de práctica clínica y revisiones. Resultados: Se seleccionaron seis artículos que revelaron que la enfermera reconoce la importancia de aplicar el proceso de enfermería, pero alega falta de tiempo debido a la sobrecarga de trabajo para su implementación. Consideraciones finales: se percibió una brecha en las publicaciones en cuanto al tema de la aplicación de la sistematización del cuidado de enfermería en el centro quirúrgico obstétrico, hecho que corrobora el impulso de futuros estudios sobre el tema.

Palabras clave: Cuidado de enfermera; Proceso de enfermería; Centro cirúrgico; Asistencia de enfermería; Embarazo.

\section{Introdução}

Embora a gestação seja um processo fisiológico, requer certos cuidados para um desfecho favorável para o binômio gestante/concepto. O enfermeiro é legal e cientificamente habilitado para acompanhar o período gravídico através da consulta de enfermagem no pré-natal e assistencial quando do trabalho de parto sem distocia. A enfermagem obstétrica atua preservando os preceitos da atenção humanizada com valorização da singularidade e protagonismo da gestante o que contribui para uma assistência livre de intervenções desnecessárias fomentando a redução da mortalidade e morbidade neonatal e materna (Cassiano et al., 2021).

Porém, nem sempre uma gestação evolui para um parto fisiológico podendo resultar na necessidade de cesariana. Dentre os agravos que podem levar a não realização de parto natural estão a pré-eclâmpsia, desproporção cefálio-pélvica, placenta prévia, malformações que impedem a passagem do concepto pelo canal vaginal, infecções com alto risco de transmissão vertical pelo contato com as secreções maternas, sofrimento fetal dentre outras (Silva et al., 2019). Neste cenário, surge a figura do enfermeiro do Centro Cirúrgico Obstétrico (CCO) que deverá prestar assistência antes, durante e após o procedimento cirúrgico.

O profissional que atua no CCO tem fundamental importância na segurança do processo transoperatório assumindo funções gerenciais e assistenciais (Soares et al., 2015). As funções gerenciais envolvem a previsão e provisão de materiais e a supervisão da equipe técnica (Martins \& Dall'agnol, 2016). Quanto as atividades assistenciais, estas devem ser pautadas na Sistematização da Assistência de Enfermagem (SAE) que faz parte do Processo de Enfermagem. No contexto perioperatório, é denominado Sistematização da Assistência de Enfermagem Perioperatória (SAEP). 
Segundo a Resolução 358/2009 do Conselho Federal de Enfermagem, a SAE possui cinco etapas interdependentes e interrelacionadas que fundamentam o cuidado de enfermagem: 1- Histórico de Enfermagem, 2- Diagnóstico de Enfermagem, 3- Planejamento, 4- Implementação e 5- Avaliação. Sua realização deve ser concretizada em todos os ambientes onde haja assistência de enfermagem. Porém, tal prática é incipiente e, por vezes, ocorre de forma fragmentada (Santos et al., 2014).

O Processo de Enfermagem (PE) proporciona autonomia ao enfermeiro, contudo alguns profissionais o veem como mera atividade burocrática não dando importância à sua relevância na prática assistencial. Outros reconhecem a importância, mas não aplicam por imperícia e há os que dominam o assunto, mas não aplicam pela sobrecarga de trabalho (Santos et al., 2014).

Justifica-se a realização deste estudo visando contribuir para sensibilização do profissional enfermeiro sobre a importância da implementação da SAE explorando as evidências científicas sobre a assistência de enfermagem à gestante no CCO instrumentalizada pelo PE contribuindo para o gerenciamento do cuidado, fomentando a translação do conhecimento.

Diante do exposto, realizou-se uma revisão integrativa para responder a seguinte pergunta norteadora: quais as evidências científicas sobre assistência de enfermagem prestada à gestante no CCO fundamentada no Processo de Enfermagem com a utilização da SAE?

O objetivo do presente estudo é investigar as evidências científicas sobre a assistência de enfermagem à gestante no centro cirúrgico obstétrico com destaque para implementação do Processo de Enfermagem.

\section{Metodologia}

Foi produzida uma Revisão Integrativa da Literatura (RIL) que corresponde a síntese do conhecimento sobre determinado assunto utilizando rigor metodológico sendo desenvolvida em seis etapas: definição da pergunta norteadora, busca e seleção dos estudos, extração dos dados relevantes, avaliação dos artigos selecionados, síntese dos resultados e apresentação da revisão (Mendes et al., 2019).

A pergunta norteadora foi construída por meio da estratégia PICo (WAH, 2014) onde P corresponde à população (gestante), I é a intervenção (aplicação da sistematização da assistência de enfermagem) e Co é referente ao contexto (assistência de enfermagem no Centro Cirúrgico Obstétrico/CCO) da junção da estratégia surgiu a pergunta de pesquisa: quais as evidências científicas sobre assistência de enfermagem prestada à gestante no CCO fundamentada no Processo de Enfermagem com a utilização da SAE?

A coleta dos dados ocorreu nas bases de dados Literatura Latino Americana e do Caribe em Ciências da Saúde (LILACS), Base de Dados de Enfermagem (BDENF) e Scientif Eletronic Library Online (SciELO). Foram utilizados descritores em saúde controlados e não controlados combinados entre si com a utilização dos operadores booleanos AND e OR (gestante OR gestação de alto risco OR gravidez AND processo de enfermagem OR sistematização da assistência de enfermagem AND centro cirúrgico OR centro cirúrgico obstétrico). Para as bases de dados LILACS e BDENF foi utilizado o Portal da Biblioteca Virtual em Saúde (BVS) e para SciELO, o site www.scielo.org. A busca ocorreu aos pares no período de agosto a setembro de 2021.

Os critérios de inclusão adotados foram: estudo primário, disponibilidade na íntegra gratuitamente, recorte temporal de cinco anos (2016-2020), idiomas inglês e português. Foram excluídas revisões, teses, dissertações, manuais, monografias, editoriais e guias de práticas clínicas. O fluxograma da seleção dos estudos consta na figura 1.

Os estudos foram analisados quanto ao conteúdo e nível de evidência proposto por Melnyk e Fineout (2005) que classificam os estudos em sete níveis à saber- I- revisões sistemáticas, metanálise de ensaios clínicos; II- ensaio clínico randomizado; III- ensaio clínico sem randomização; IV- coorte, caso-controle; V- revisão sistemática de trabalhos descritivos; VI- único estudo descritivo ou qualitativo; VII- opinião de comitês de especialistas ou autoridades. 
Figura 1 - Fluxograma de seleção dos artigos.

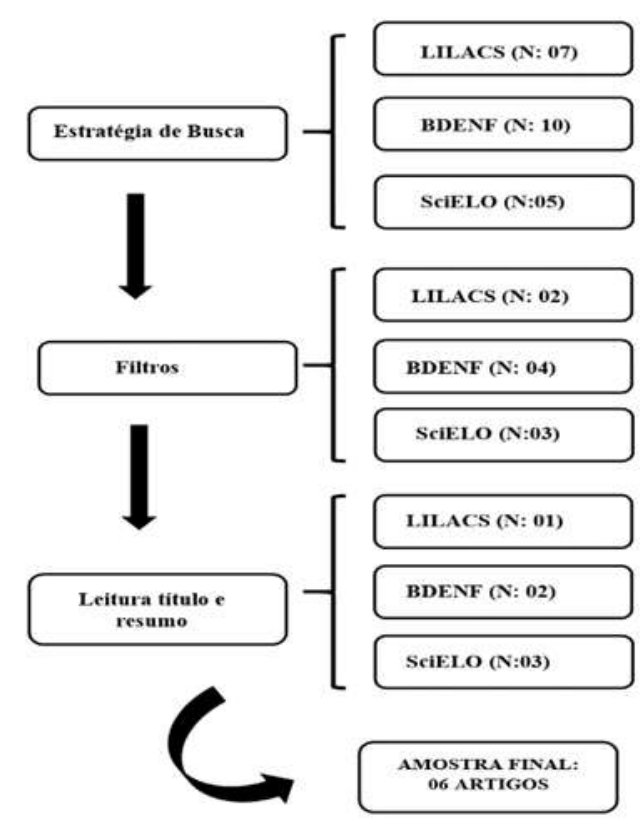

Fonte: Elaborado pelos autores. Brasil, Manaus (2022).

A extração dos dados ocorreu com a utilização de protocolo elaborado pelos autores onde os dados foram sintetizados e catalogados em tabela do programa Microsoft Excel. Por tratar-se de uma revisão integrativa da literatura, não foi necessária anuência por Comitê de Ética em Pesquisa.

\section{Resultados e Discussão}

Considerando os critérios de inclusão e exclusão e a estratégia de busca adotadas, não foi possível encontrar artigos sobre a temática em específico: assistência de enfermagem à gestante no CCO com implementação da SAE. Os estudos que emergiram tratavam da SAE no contexto de condições clínicas específicas sem relação com CCO. Partindo dessa lacuna, optou-se por selecionar artigos que abordassem a SAE enquanto processo instrumentalizador do trabalho do enfermeiro, considerando os estudos revelados por meio da estratégia de busca eleita neste estudo.

Portanto, da busca inicial foram encontrados 22 estudos. Com aplicação dos critérios de elegibilidade remanesceram 09 e da leitura do resumo e título permaneceram 06 dos quais foram selecionados para amostra final (Quadro 01), pois faziam referência a SAE enquanto ordenadora do cuidado/assistência de enfermagem.

Os estudos selecionados estão indexados nas bases de dados: um na LILACS, dois na BDENF e três na SciELO. Sendo cinco estudos descritivos e um de reflexão. Quanto ao nível de evidência, cinco possuem nível VI (A1, A2, A3, A4 e A5) e um com nível VII (A6) denotando evidência incipiente conforme a referência adotada (Melnyk \& Fineout, 2005). 
Quadro 1 - Sumarização dos estudos selecionados.

\begin{tabular}{|c|c|c|c|c|c|}
\hline & $\begin{array}{c}\text { Autores } \\
\text { Ano } \\
\text { País } \\
\end{array}$ & Título & Base de dados & $\begin{array}{l}\text { Tipo de estudo e } \\
\text { Nível de } \\
\text { Evidência } \\
\end{array}$ & Resultados \\
\hline 1 & $\begin{array}{c}\text { Azevedo et al } \\
2019 \\
\text { Brasil }\end{array}$ & $\begin{array}{lrr}\begin{array}{l}\text { Documentação } \\
\text { enfermagem }\end{array} & \text { do } & \text { processo de } \\
\text { públicas de saúde. } & \text { instituições } \\
\text { púb } & \\
\end{array}$ & LILACS & $\begin{array}{l}\text { Descritivo } \\
\text { VI }\end{array}$ & $\begin{array}{l}\text { O PE é documentado, porém nem } \\
\text { sempre conforme as exigências legais. }\end{array}$ \\
\hline A2 & $\begin{array}{c}\text { Ribeiro et al } \\
2017 \\
\text { Brasil }\end{array}$ & $\begin{array}{l}\text { Atitudes dos enfermeiros de centro } \\
\text { cirúrgico diante da sistematização } \\
\text { da assistência de enfermagem } \\
\text { perioperatória. }\end{array}$ & BDENF & $\begin{array}{l}\text { Descritivo } \\
\text { VI }\end{array}$ & $\begin{array}{l}\text { A SAE é um desafio para o enfermeiro } \\
\text { que atua no centro cirúrgico onde sua } \\
\text { aplicação é fragmentada. }\end{array}$ \\
\hline A3 & $\begin{array}{c}\text { liveira et al } \\
2016 \\
\text { Brasil }\end{array}$ & $\begin{array}{l}\text { Diagnósticos, resultados e } \\
\text { intervenções de enfermagem para } \\
\text { pacientes da clínica cirúrgica de um } \\
\text { hospital escola. }\end{array}$ & BDENF & $\begin{array}{l}\text { Descritivo } \\
\text { VI }\end{array}$ & $\begin{array}{l}\text { Os profissionais sentem dificuldade em } \\
\text { identificar diagnósticos, resultados e } \\
\text { intervenções }\end{array}$ \\
\hline A4 & $\begin{array}{c}\text { Barreto et al } \\
2020 \\
\text { Brasil }\end{array}$ & $\begin{array}{l}\text { Sistematização da assistência de } \\
\text { enfermagem: a práxis do enfermeiro } \\
\text { de hospital de pequeno porte }\end{array}$ & SciELO & $\begin{array}{l}\text { Descritivo } \\
\text { VI }\end{array}$ & $\begin{array}{l}\text { É preciso promover estratégias que } \\
\text { permitam aos enfermeiros se } \\
\text { apoderarem e aplicarem a SAE no } \\
\text { contexto hospitalar. }\end{array}$ \\
\hline A6 & $\begin{array}{l}\text { Gutiérrez \& } \\
\text { Morais } \\
2017 \\
\text { Brasil }\end{array}$ & $\begin{array}{l}\text { Sistematização da Assistência } \\
\text { de Enfermagem e a formação da } \\
\text { identidade profissional. }\end{array}$ & SciELO & $\begin{array}{l}\text { Estudo de reflexão } \\
\text { VII }\end{array}$ & $\begin{array}{l}\text { A SAE é um desafio, pois somente } \\
\text { quando atribuímos significado ao que } \\
\text { sabemos e fazemos é que incorporamos } \\
\text { como algo próprio à nossa identidade } \\
\text { profissional. }\end{array}$ \\
\hline
\end{tabular}

Fonte: Autores.

O estudo A1(Azevedo et al., 2019) propôs a identificação da prevalência de documentação do PE em hospitais e ambulatórios considerando sua obrigatoriedade legal. Ratifica a importância da realização da SAE para cientificidade da profissão e melhoria da comunicação entre os profissionais contribuindo para integralidade do cuidado. Concluiu que a documentação do processo ocorre de forma fragmentada onde quanto menor o tempo de contato com o usuário/cliente, mais frágil é o registro. Ressalta que para concretização contínua do PE, se faz necessário a reorganização das práticas em saúde.

Corroborando com o estudo de Azevedo et al. (2019), o artigo A2 (Ribeiro et al., 2017) afirma que a SAE, quando utilizada durante o período perioperatório, é realizada com fragmentação das etapas. O objetivo do estudo foi descrever as atitudes do profissional enfermeiro diante da realização da SAE no centro cirúrgico. Foi evidenciado que $70 \%$ dos participantes do estudo $(n=14)$ a utilizavam pouco ou nenhum momento e $90 \%(n=18)$ não procuravam participar de cursos sobre a temática. Destaca que o enfermeiro reconhece o processo como significativo para a prática assistencial, porém há considerado déficit teórico quanto ao tema o que implica diretamente em sua implementação. Dentre os estudos selecionados, foi o que mais se aproximou do objetivo deste estudo.

Identificar possíveis diagnósticos, intervenções e resultados para colaborar com a implementação das etapas da SAE voltadas aos usuários hospitalizados em clínicas cirúrgicas foi o objetivo do estudo A3 (Oliveira et al., 2016). Identificou-se que os profissionais têm dificuldade em formalizar a SAE. Foram elencados diversos diagnósticos de enfermagem (DE) com suas respectivas intervenções e resultados baseados na teoria das necessidades humanas básicas de Wanda Horta. Dentre os DE definidos, destacam-se: ansiedade, risco para infecção, sono e repouso prejudicados. Em consonância aos estudos A1 e A2, este conclui que o PE favorece e organiza o cuidado de enfermagem.

$\mathrm{O}$ apoio dos gestores, educação permanente dos profissionais de enfermagem e a padronização de instrumentos são imprescindíveis para que a SAE seja uma realidade na vivência assistencial dos enfermeiros, é o que afirma o estudo A4 (Barreto et al., 2020). Objetivou-se nesse estudo, descrever a atuação dos enfermeiros (em unidade hospitalar) relacionada à SAE. Concluiu-se que a sobrecarga de trabalho e a falta de apoio institucional dificultam sua operacionalização. Contudo, os 
enfermeiros que reconhecem a importância do PE utilizam estratégias para sanar as dificuldades enfrentadas. À exemplo: priorização dos pacientes mais graves, quando da utilização da sistematização, sem fragmentação das etapas do processo.

Através do estudo A5 (Oliveira et al., 2019) foi possível conhecer a percepção e o conhecimento dos profissionais de enfermagem a respeito da SAE. Foi constatado que seu uso está diretamente relacionado ao nível de formação de cada indivíduo e que todos são cientes da obrigatoriedade e importância do PE, mas não utilizam por baixo conhecimento sobre a temática. Fato que reforça o exposto no estudo A4 quanto a necessidade de educação permanente desses profissionais, pois é através da sistematização do cuidado que o mesmo será gerido em sua integralidade.

Para o fortalecimento de nossa identidade profissional, é necessário compreender os elementos significativos do PE e da SAE considerando suas potencialidades e dificuldades para que seja viável sua implementação plena, essa é a inferência do estudo A6 (Gutiérrez \& Morais, 2017). O texto nos remete a uma reflexão sobre o fazer/ saber de nossa prática relatando a necessidade de integração e diálogo entre os envolvidos no cuidar: entidades de classe, profissionais, usuários e gestores.

Os seis estudos selecionados são unânimes em apontar a sobrecarga de trabalho, déficit de conhecimento, falta de apoio institucional e a fragmentação da SAE como fatores que dificultam sua operacionalização. Bem como, trazem a informação que os profissionais reconhecem a importância da utilização do PE para a valorização e cientificidade da profissão; mas não a utilizam por falta de preparo científico e/ou pelas demandas administrativas institucionais que são delegadas concomitantemente aos cuidados clínicos.

Segundo Soares et al. (2015), esses vieses para consolidação da SAE podem ser amenizados com o aperfeiçoamento dos serviços através do apoio institucional na oferta de condições concretas que facilitem sua operacionalização tais como: instituição de protocolos, dimensionamento adequado dos recursos humanos, capacitação dos profissionais e oferta de impressos padronizados.

De acordo com estudo realizado por Terto et al.(2021), a internação precoce da gestante ocasiona intervenções obstétricas desnecessárias que culmina com a indicação de cesariana. Nesse contexto, surge o cuidado de enfermagem cirúrgico que envolve desde o manejo clínico à execução de atividades educativas (Vicente et al., 2021). Através do uso da SAE esses cuidados são ofertados de forma individualizada conferindo uma assistência integral e resolutiva. Contudo, nem sempre é possível sua utilização (Ribeiro et al., 2017).

O uso da sistematização da assistência no ambiente cirúrgico confere segurança ao paciente, precisão dos registros e facilita a comunicação entre a equipe sendo possível o acompanhamento gradativo e integral dos pacientes (Lopes et al.,2015). Assim como em outros pontos da assistência, sua execução é permeada por dificuldades decorrentes do despreparo quanto ao conhecimento teórico gerando insegurança em sua aplicação e promovendo um cuidado tecnicista (Santos et al., 2017). Portanto, o profissional deve empoderar-se de sua prática buscando aprimorar seus conhecimentos sobre a SAE para a excelência da assistência prestada.

Reconhecer as fragilidades da efetivação da SAE não pode ser justificativa plena para sua não aplicação. Tais fragilidades dificultam, mas não impossibilitam sua concretização. É necessária mudança de comportamento dos profissionais e, principalmente, organizacional para que essas barreiras sejam vencidas (Santos et al., 2018). É relevante ressaltar o papel das instituições de ensino no fomento à aplicação do PE, sensibilizando os futuros profissionais quanto a existência desses desafios e fazendo-os pensar criticamente sobre estratégias para superá-los (Souza et al., 2015).

Este estudo limita-se pela lacuna encontrada quanto à sua temática central. Contudo, é relevante para deixar à mostra que os desafios e potencialidades do PE e da SAE no CCO não são apenas relacionados à prática profissional. Mas, também, quanto às publicações sobre o assunto. Espera- se que o trabalho aqui desenvolvido possa ser impulsionador de futuras publicações. 


\section{Considerações Finais}

Embora os achados deste trabalho tenham convergido para explicitação de lacuna quanto ao seu objetivo, foi relevante dar continuidade para fomentar a necessidade de futuras publicações sobre a temática: "Sistematização da assistência de enfermagem à gestante no CCO”. É possível que a inclusão de outras bases de dados possa ampliar os resultados, contudo para esse trabalho optou-se por manter as elencadas inicialmente. Estratégias como informatização do processo, capacitação dos profissionais e adequação e/ou disponibilidade de instrumentos padronizados podem facilitar a aplicação da SAE em sua integralidade. Desafios existem e sempre existirão em qualquer profissão. Porém, o comprometimento individual é o diferencial para a eficácia do trabalho que se pretende desenvolver e, consequentemente, superar os desafios. Dentre as potencialidades da execução do PE pela SAE podemos citar a valorização e o reconhecimento cientifico das atribuições e competências da equipe de enfermagem seja por ela mesmo, como pelos outros profissionais de saúde. Espera-se que novas publicações sejam produzidas considerando os achados/lacunas desse estudo, visando a efetivação da translação do conhecimento.

\section{Referências}

Azevedo, O. A., Guedes, E. S., Araújo, S. A. N., Maia, M. M. \& Cruz, D. A. L. M. (2019). Documentação do processo de enfermagem em instituições públicas de saúde. Revista da Escola de Enfermagem da USP, 53, e03471. https://doi.org/10.1590/S1980-220X2018003703471.

Barreto, M. S., Prado, E., Lucena, A. C. R. M., Rissardo, L. K., Furlan, M. C. R. \& Marcon, S. S. (2020). Sistematização da assistência de enfermagem: a práxis do enfermeiro de hospital de pequeno porte. Escola Anna Nery, 24 (4), p. e20200005. https://doi.org/10.1590/2177-9465-EAN-2020-0005.

Cassiano, A. N., Menezes, R. M. P., Medeiros, S. M., Silva, C. J. A. \& Lima, M. C. R. A. (2021). Atuação do enfermeiro obstétrico na perspectiva das epistemologias do Sul. Escola Anna Nery, 25(1), e20200057. https://doi.org/10.1590/2177-9465-EAN-2020-0057.

Gutiérrez, M. G. R. \& Morais, S. C. R. V. (2017). Sistematização da assistência de enfermagem e a formação da identidade profissional. Revista Brasileira de Enfermagem, 70 (2), 436-441. https://doi.org/10.1590/0034-7167-2016-0515.

Lopes, N. P., Gaspariny, C. \& Koller, F. J. (2015). A importância da assistência de enfermagem perioperatória como principal mecanismo de atuação do enfermeiro com foco na fase pré-operatória. REVENF, 4. Nhttps://unisantacruz.edu.br/revistas/index.php/Revenf/article/view/1042.

Martins, F. Z. \& Dall'agno, L. C. M. (2016). Centro cirúrgico: desafios e estratégias do enfermeiro nas atividades gerenciais. Revista Gaúcha de Enfermagem, 37 (4), e56945, 2016. https://doi.org/10.1590/1983-1447.2016.04.56945.

Melnyk, B. M. \& Fineout, O. E. (2005). Making the case for evidence-based practice. In: Melnyk BM, Fineout-Overholt E. Evidence based practice in nursing $\&$ healthcare. A guide to best practice. Lippincot Williams \& Wilkins.

Mendes, K. D. S., Silveira, R. C. C. P., Galvão, C. M. (2019). Uso de gerenciador de referência bibliográfica na seleção de estudos primários em revisões integrativas. Texto \& Contexto - Enfermagem, 28, e20170204. https://doi.org/10.1590/1980-265X-TCE-2017-0204.

Oliveira, M. R., Almeida, P. C. A., Moreira, T. M. M. \& Torres, R. A. M. (2019). Sistematização da assistência de enfermagem: percepção e conhecimento da enfermagem brasileira. Revista Brasileira de Enfermagem, 72(6), 1547-1553. https://doi.org/10.1590/0034-7167-2018-0606.

Oliveira, D. M., Vieira, M. M., Carvalho, M. W. A. \& Nóbrega, M. M. L. (2016). Diagnósticos, resultados e intervenções de enfermagem para pacientes da clínica cirúrgica de um hospital escola. JNUOL, 10(12). https://periodicos.ufpe.br/revistas/revistaenfermagem/article/view/11518.

Ribeiro, E., Ferraz, K. M. C. \& Duran, E. C. M. (2017). Atitudes dos enfermeiros de centro cirúrgico diante da sistematização da assistência de enfermagem perioperatória. Revista SOBECC, 22(4), 201-207. https://revista.sobecc.org.br/sobecc/article/view/231.

Santos, S. M., Braquehais, A. R. \& Abreu, R. N. D. C. (2018). Desafios e potencialidades na implementação da sistematização da assistência de enfermagem a pacientes críticos. ReTEP, 10(3), 9-13. http://www.coren-ce.org.br/wp-content/uploads/2019/11/Desafios-e-potencialidades-naimplementa\%C3\%A7\%C3\%A3o-da-Sistematiza\%C3\%A7\%C3\%A3o-da-Assist\%C3\%AAncia-de-Enfermagem-a-pacientes-cr\%C3\%ADticos.pdf.

Santos, N. P., Campos, C. A. R. \& Gonzaga, M. F. N. (2017). Dificuldades na implementação da sistematização de enfermagem. Revista Saúde em Foco, n 9. https://portal.unisepe.com.br/unifia/wp-content/uploads/sites/10001/2018/06/048_dificuldades.pdf.

Santos, W. N., Santos, A. M. S., Lopes, T. R. P. S. L., Madeira, M. Z. A. \& Rocha, F. C. V. (2014). Sistematização da Assistência de Enfermagem: o contexto histórico, o processo e obstáculos da implantação. JMPHC | Journal of Management \& Primary Health Care, 5(2), 153-158, https://doi.org/10.14295/jmphc.v5i2.210.

Silva, A. P. Romero, R. T., Bragantine, A., Barbieri, A. A. D. M. \& Lago, M, T. G. (2019).As indicações de cesáreas no Brasil: uma revisão de literatura integrativa. Revista Eletrônica Acervo Saúde, n. 24, e624. https://acervomais.com.br/index.php/saude/article/view/624.

Soares, M. I., Soares, M. I., Resck, Z. M. R., Terra, F. S. \& Camelo, S. H. H. (2015). Sistematização da assistência de enfermagem: facilidades e desafios do enfermeiro na gerência da assistência. Escola Anna Nery, 19(1), 47-53. https://doi.org/10.5935/1414-8145.20150007. 
Research, Society and Development, v. 11, n. 1, e14111125034, 2022

(CC BY 4.0) | ISSN 2525-3409 | DOI: http://dx.doi.org/10.33448/rsd-v11i1.25034

Souza, L. P., Vasconcelos, C. \& Parra, V. (2-15). Processo de enfermagem: dificuldades enfrentadas pelos enfermeiros de um hospital público de grande porte na Amazônia, Brasil. Brazilian journal of surgery and clinical research/BJSCR, 10(1), 05-20. https://www.mastereditora.com.br/periodico/20150304_162920.pdf.

Terto, R. L., Silva, T. P. R., Viana, T. G. F., Sousa, A. M. M., Martins, E. F., Souza, K. V. \& Matozinhos, F. P. (2021) Associação entre internação precoce de gestantes e uso de intervenções obstétricas e cesarianas: estudo transversal. Rev Bras Enferm, 74(4), e20200397. https://doi.org/10.1590/0034-7167-2020-0397

Vicente, C., Amante, L. N., Sebold, L. F., Girondi, J. B. R., Martins, T., Salum, N. C. \& Maia, A. R. C. R. (2021). Associação entre internação precoce de gestantes e uso de intervenções obstétricas e cesarianas: estudo transversal. Cogitare enferm, v26, e72640. https://www.scielo.br/j/cenf/a/CygBHbq4TsFsccGtSw4ZcLf/?format=pdf\&lang=pt.

WAH. (2014). O que é PICO e PICo? https://pt.slideshare.net/bibliotecaee/o-que-pico-e-pico. 